\title{
ОСОБЕННОСТИ ПРОЕКТИРОВАНИЯ ВЕТРОГЕНЕРАТОРОВ ПОВЫШЕННОЙ МОЩНОСТИ (600 $\div 3600)$ КВТ С ВОЗБУЖДЕНИЕМ ОТ ПОСТОЯННЫХ МАГНИТОВ
}

\author{
Ю.Н. Перминов ${ }^{1}$, канд. техн. наук, Е.А. Монахов², Л.П. Волков ${ }^{3}$ \\ ${ }^{1}$ Институт возобновляемой энергетики НАН Украины, \\ 02094, ул. Гната Хоткевича, 20А, г. Киев, Украина. \\ ${ }^{2}$ Национальный технический университет Украины «Киевский политехнический институт имени Игоря Сикорского», \\ 03056, пр. Победы, 36, г. Киев, Украина. \\ ${ }^{3}$ Институт экологического управления и сбалансированного природопользования, \\ 02002, ул. Никольско-Слободская, 6-Д, г. Киев, Украина.
}

В основу работы положены результаты расчетов ряда синхронных безредукторных ветрогенераторов модульных конструкиий [1] с возбуждением от постоянных магнитов NdFeB мощеностью $(600 \div 3600)$ кBт при принятой за рубежом расчетной скорости ветра - 12,5 м/с. Такой подход исследования машин позволяет оиенить сложную взаимосвязь их параметров при минимуме допущений, в частности, учесть насыщение наиболее нагруженных участков магнитной цепи и определить иелесообразность применения некоторых возможных вариантов обмоток, которые обеспечивают наибольшую электромагнитную мощность генераторов с учетом технологичности их выполнения; показаны особенности изменения зубиовой зоны машин повышенной мощности, обусловленные низкой скоростью вращения генераторов 6 безредукторных установках и обоснована по этой причине возможность применения "меандровой" обмотки.

В такой обмотке каждая фаза выполняется в виде одного непрерывного проводника, что позволяет исключить соединение секиий в фазе, имеющее место в традиционных обмотках, уменьшить сопротивление обмотки благодаря отсутствию одной лобовой части в сравнении с витком секиии. При решении технологии изготовления таких обмоток в виде шинь возможно повышение коэффиииента заполнения пазов и мощности генератора в определенных габаритах. Возможно изготовление такой обмотки кабелями, в этом случае трехфазная обмотка будет представлять три кабеля, что позволит упростить её изготовление, но коэффициент заполнения пазов будет меньше.

В работе проведено сравнение различных вариантов меандровых обмоток с иелью упрощения их изготовления $и$ традиционных всыпных обмоток с различным количеством пазов на полюс и фазу с иелью увеличения зубиового шага и упрощения изготовления пакета статора и обмотки. Библ. 9, табл. 1, рис. 5.

Ключевые слова: безредукторные генераторы модульной конструкиии, варианты обмоток, зубиовая зона, "меандровая обмотка", коэффициент заполнения пазов, число пазов на полюс и фазу, число витков в секции, активная длина проводников, электромагнитная мощность.

\section{FEATURES OF DESIGN THE PERMANENT MAGNET WINDGENERATOR OF HIGH POWER $(600 \div 3600) \mathrm{kW}$}

\author{
Y. Permynov ${ }^{1}$, candidate of technical sciences, E. Monakhov ${ }^{2}$, L. Volkov ${ }^{3}$ \\ ${ }^{1}$ Institute of Renewable Energy of the National Academy of Sciences of Ukraine, \\ 02094, 20A Hnata Khotkevycha St., Kyiv, Ukraine. \\ ${ }^{2}$ National Technical University of Ukraine «Igor Sikorsky Kyiv Polytechnic Institute», \\ 03056, 37, Peremohy av., Kyiv, Ukraine. \\ ${ }^{3}$ Institute of ecological management and balanced nature management, \\ 02002, 6-D, Nikolsko-Slobidska Str., Kyiv, Ukraine.
}

The paper is based on the results of calculations of a number of synchronous gearless direct-drive wind generators of modular design [1] with permanent magnet excitation in range of power since 600 to $3600 \mathrm{~kW}$ at a wind speed that is taken abroad during the design $-12.5 \mathrm{~m} / \mathrm{s}$. This approach to the study of machines allows to evaluate the complex relationship of their parameters with a minimum of assumptions, in particular, to take into account the saturation of the most loaded sections of the magnetic circuit and determine the feasibility of using some possible winding options that provide the greatest electromagnetic power to the generators, taking into account the manufacturability of their implementation; the features of the change in the tooth zone of high-power machines are shown, due to the low rotation speed of the generators in gearless installations and justified for this reason, the possibility of using the "meander" winding.

In such type of winding each phase is made in the form of a single continuous conductor, which eliminates the connection of sections in phase, which takes place in traditional windings, and reduces the resistance of the winding due to the absence of one frontal part in comparison with the coil section. When solving the manufacturing technology of such windings in the form of a copper bus, it is possible to increase the fill factor of the slots and the power of the generator in certain dimensions. It is possible to manufacture such a winding of cables, in this case the three-phase winding will represent three cables, which will simplify its manufacture, but the fill factor of the slots will be less.

In the paper different options of meander windings are compared in order to simplify their manufacture and traditional loose windings with a different number of slots per pole and phase in order to increase the tooth pitch and simplify the manufacture of the stator core and winding. Ref. 9, tabl. 1, fig. 5 .

Keywords: gearless generators of modular design; winding options; tooth zone; meander winding; slot fill factor; the number of slots per pole and phase; the number of turns in the section; active length of conductors; electromagnetic power.

(C) Ю.Н. Перминов, Е.А. Монахов, Л.П. Волков, 2020

Відновлювана енергетика. 2020. № 2 

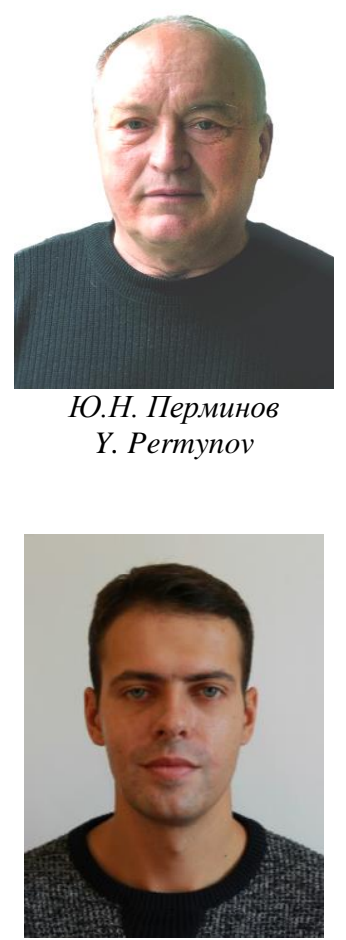

E.A. Монахов

E. Monakhov

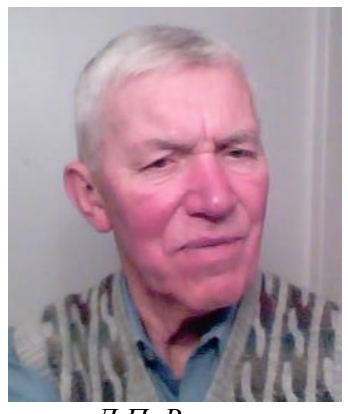

Л.П. Волков

L. Volkov
Сведения об авторе: канд. техн. наук, старший научный сотрудник Института возобновляемой энергетики НАН Украины. Образование: окончил в 1968 г. Киевский политехнический институт по специальности «Электрические машины и аппараты». Научная сфера: ветрогенераторы, ветроустановки малой мощности, гидрогенераторы, устройства с постоянными магнитами.

Публикации: 88 .

ORCID: $10000-0001-5604-8327$

Контакты: Тел./факс +38-044-206-28-09

e-mail: renewable@ukr.net

Сведения об авторе: асистент кафедры электромеханики КПИ им. И. Сикорского, Киев.

Образование: окончил в 2012 г. Киевский политехнический институт по специальности «Электрические машины и аппараты». Научная сфера: машины с постоянными магнитами, синхронные генераторы, малые электростанции, гидроэлектростанции. Публикации: 27.

ORCID: 0000-0001-8408-8051

Контакты: тел./факс: +38-095-317-61-15

e-mail: emonachov@gmail.com

Сведения об авторе: старший научный сотрудник отдела научно-методологического обеспечения Института экологического управления и сбалансированного природопользования, г. Киев.

Образование: окончил в 1959 г. Московский энергетический институт (МЭИ) по специальности «Электрификация промышленных предприятий».

Научная сфера: электропривод, электрические машины.

Публикации: 21.

ORCID: 0000-0003-1988-9666

Контакты: тел/факс +38-044-206-28-09

e-mail: volleo35@gmail.com
Author information: candidate of technical sciences, Senior Researcher at Institute for Renewable Energy, National Academy of Sciences of Ukraine.

Education: graduated from the Kiev Polytechnic Institute in 1968 with a degree in Electrical Machines and Apparatuses.

Research area: wind power systems, small capacity wind units, hydrogenerators, devices with permanent magnets

Publications: 88.

ORCID: $10000-0001-5604-8327$

Contacts: phone./fax: +38-044-206-28-09

e-mail: renewable@ukr.net

Author information: assistant of department of electomechanics in Igor Sikorsky Kyiv Polytechnic Institute.

Education: graduated from the Kyiv Polytechnic Institute in 2012 with the specialization «Electrical machines and apparatus».

Research area: permanent magnet machines, synchronous generators, small energy plants, hydropower plants.

Publications: 27.

ORCID: 0000-0001-8408-8051

Contacts: phone./fax: $+38-095-317-61-15$

e-mail: emonachov@gmail.com

Author information: senior staff scientist of scientific-methodological support department in Institute of ecological management and balanced nature management, Kiev.

Education: graduated the Moscow energy institute in 1959 with specialization «Electrification of industrial enterprises».

Research area: electric drive, electrical machines.

Publications: 21.

Contacts: phone./fax +38-044-206-28-09

e-mail: volleo35@gmail.com

\section{Перечень используемых обозначений и сокращений:}

$M$ - электромагнитный момент;

$B_{\delta} \quad$ - индукция в рабочем зазоре;

$A$ - линейная нагрузка;

$q$ - количество пазов на полюс и фазу;

$k_{3} \quad$ - коэффициент заполнения паза;

$P \quad$ - мощность генератора;

$\rho \quad$ - плотность воздуха;

$S \quad$ - площадь ометаемой поверхности;

$D_{a} \quad$ - диаметр ротора;

$l_{a}$ - активная длина ротора;

$\rho \quad$ - плотность воздуха;

$v \quad$ - скорость воздушного потока;

$k$ - К.П.Д. ветроколеса;

$D \quad$ - диаметр ветроколеса;

$\varpi \quad$ - угловая частота вращения;

$S$ - площадь ометаемой поверхности; $z^{\prime} \quad$ - коэффициент быстроходности;

$\alpha_{i} \quad$ - коэффициент полюсного перекрытия;

$\lambda$ - отношение длины ротора к диаметру;

$p^{\prime} \quad$ - число пар полюсов;

$f \quad$ - частота напряжения;

$z \quad$ - число пазов;

m - число фаз;

$t_{z} \quad$ - зубцовый шаг;

$z_{p} \quad$ - число пазов на полюс и фазу;

$\Phi_{\delta} \quad$ - полезный поток;

$W_{\phi} \quad$-число витков в фазе;

$k_{o}$ - обмоточный коэффициент;

$l_{c p}$ - активная длина проводников фазы;

$v \quad$ - линейная скорость; 


$$
\begin{array}{ll}
E_{\phi} & \text { - электродвижущая сила фазы; } \\
U_{\phi} & \text { - фазное напряжение; } \\
j & \text { - плотность тока; } \\
q_{\phi} & \text { - сечение провода; }
\end{array}
$$

Введение. Отличительная ветрогенераторов при

особенность отсутствии мультипликаторов - низкая частота вращения, обусловленная частотой вращения ветроколеса [2]. С увеличением мощности ветроустановки от $(600 \div 3600)$ кВт частота вращения ветроколеса уменьшается соответственно от 80 до 22 об/мин.

Величина э.д.с., наводимая в обмотке генератора, пропорциональна частоте вращения ротора. С уменьшением частоты вращения одна и та же мощность может быть получена за счет роста электромагнитного момента $\left(M_{\ni}=\frac{P}{\varpi}\right)$, который определяется произведением объема

$\begin{array}{ll}S_{n} & \text { - площадь паза; } \\ \cos \varphi & \text { - коэффициент мощности; } \\ b_{z} & \text { - ширина зубца; } \\ b_{n} & \text { - ширина паза. }\end{array}$

ротора на удельные электромагнитные нагрузки: индукцию в рабочем зазоре ( $B_{\delta}$, Тл) и линейную нагрузку ( $A, \mathrm{~A} / \mathrm{M})$. Рост объема ротора сопровождается увеличением массогабаритных показателей всей машины и падением её удельной мощности, определяемой отношением мощности к массе машины (кВт/кг). На рис.1 показана зависимость удельной мощности в зависимости от номинальной для машин с разными скоростями вращения, построенной по параметрам серийных машин, оптимизированных по габаритам и массе.

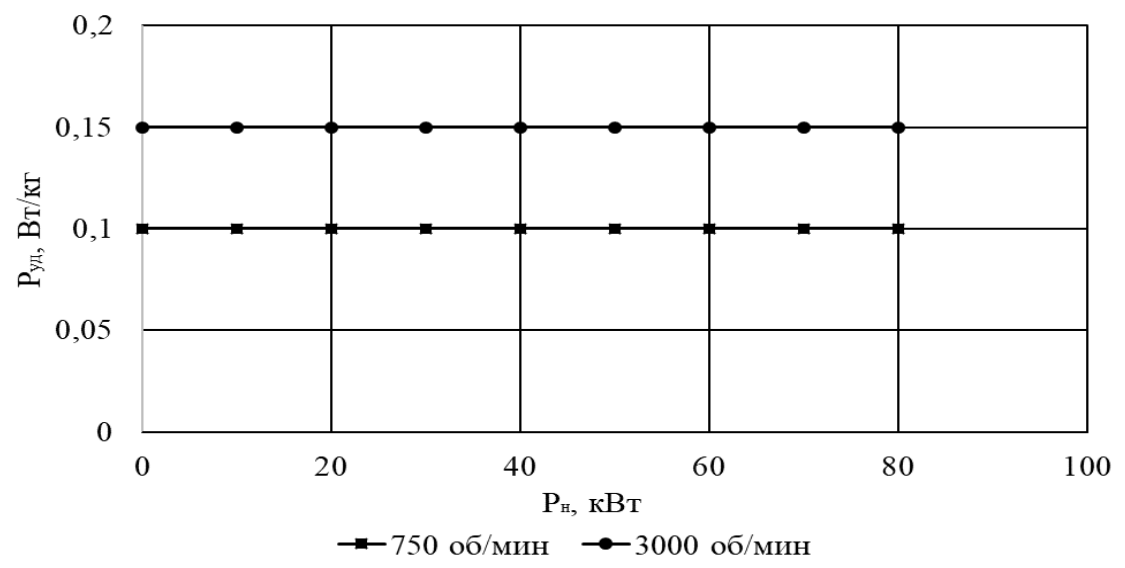

Рис. 1. Зависимость удельной мощности от номинальной при разных скоростях вращения.

Fig. 1. The dependence of the power to weigh ratio at different speed versus rated power.

Удельная электромагнитная мощность генератора также существенно зависит от геометрических соотношений зубцовой зоны, с отношения диаметра ротора и статора, коэффициента заполнения паза. При определенном (заданном) диаметре статора увеличение диаметра ротора сопровождается увеличением полного магнитного потока и уменьшением токового слоя и полного тока. Так как электромагнитный момент генератора определяется произведением магнитного потока и тока, то существует оптимальное соотношение диаметра ротора и статора.

Увеличение коэффициента заполнения паза при принятой плотности тока в проводнике тоже влияет на величину полного тока и электромагнитного момента. Коэффициент заполнения паза определяется отношением сечения проводников в пазу к всему сечению паза, и зависит от типа применённой обмотки, поэтому в этой работе проведено сравнение некоторых обмоток: двухслойной, всыпной из нескольких параллельных ветвей в фазе и нескольких параллельных проводников в секции с разным количеством пазов на полюс и фазу $(q=1 ; 0,5)$ и известным для таких обмоток ориентировочным коэффициентом заполнения (Кз $\approx$ 0,34); сравнивались варианты "меандровых" обмоток с разными коэффициентами заполнения (Кз $\approx 0,34 ; 0,6)$.

Постановка задачи. Электромагнитная мощность генератора определяется многими взаимосвязанными факторами: геометрическими соотношениями ротора и статора; допустимыми удельными нагрузками - индукцией и линейной нагрузкой $\left(A, B_{\delta}\right)$; частотой вращения и др.

Преобразование механической энергии приводного двигателя (движителя) в электрическую происходит в основном в области рабочего зазора, поэтому зависит от геометрии 
зубцового слоя статора, коэффициента заполнения пазов проводниками, типа примененной обмотки. Исследование влияния этих факторов на электромагнитную мощность генератора является целью данной работы.

При заданной скорости ветра мощность ветрового потока определяется формулой:

$$
P=\frac{\rho \cdot S \cdot v^{3} \cdot k}{2 \cdot \eta_{\text {ген }}}
$$

где $v=12,5(M / c)-$ принятая скорость ветрового потока $(\mathrm{M} / \mathrm{c}) ; \quad \rho=1,3\left(\kappa 2 / \mathrm{M}^{3}\right)-$ плотность воздуха; $v$ - скорость ветрового потока (м/с). $S$ - площадь ометаемой поверхности ветроколеса $\left(\mathrm{M}^{2}\right) ; k \approx 0,3$ - коэффициент полезного действия ветроколеса; $\eta_{\text {ген }}-$ К.П.Д. генератора.

Из (1) определяется площадь и диаметр ветроколеса (м2):

$$
D=\sqrt{\frac{S}{0,785}} .
$$

Частота вращения ветроколеса:

$$
\varpi=\frac{z^{\prime} \cdot v}{R},
$$

где $z^{\prime} \approx 4,6$ - коэффициент быстроходности ветроколеса, принят по уже изготовленным ветроустановкам.

Исходными номинальными параметрами при проектировании генераторов являются:

- мощность генератора;

- частота вращения;

- фазное напряжение;

- частота выходного напряжения;

- к.п.д. генератора.

По указанным параметрам определяется электромагнитный момент:

$$
M_{\text {э }}=\alpha_{i} \cdot A \cdot B_{\delta} \cdot \pi \cdot D_{p}^{3} \cdot \lambda(\mathrm{H} \cdot \mathrm{M})
$$

где $\alpha_{i} \approx 0,8 \quad-\quad$ коэффициент полюсного перекрытия - на основе опыта проектирования; $A \approx 10 \cdot 10^{3}(\mathrm{~A} / \mathrm{M}) \quad$ - линейная нагрузка; $B_{\delta} \approx 0,8$ (Тл) -индукция в рабочем зазоре - по опыту проектирования; $\lambda \approx 0,3$ - отношение активной длины ротора к диаметру принимается по условию агрегатирования генератора в головке ветроустановки;

Диаметр ротора:

$$
D_{p}=\sqrt[3]{\frac{M_{\text {э }}}{\alpha_{i} \cdot A \cdot B_{\delta} \cdot \pi \cdot \lambda}},(\mathrm{M})
$$

Число пар полюсов:

$$
p^{\prime}=\frac{60 \cdot f}{n},
$$

где $f=(50 ; 60)(Г ц) \quad-\quad$ частота выходного напряжения; $n$ (об/мин) - частота вращения ротора.

Число пазов (зубцов) ротора

$$
z=2 \cdot p^{\prime} \cdot m \cdot q
$$

где $m$ - число фаз; $q$ - число пазов на полюс и фазу, которое часто принимается равным единице.

Как видно из (2), (7) с увеличением мощности генератора увеличивается диаметр ротора и при заданном q - увеличивается число пазов. При этом, как показали расчеты ряда генератора, мощностью от $(600 \div 3600)$ кВт при указанных исходных параметрах генератора и ветроустановки зубцовое деление $\left(b_{z}+b_{n}\right)$ меняется несущественно:

$$
t_{z}=\frac{\pi \cdot D_{p}}{z} \approx 15(\mathrm{MM})
$$

и можно считать равенство ширины зубца и паза - оптимальным по величине электромагнитного момента, который определяется произведением магнитного потока и полного тока машины. Зубец является наиболее насыщенным участком магнитной цепи и определяет полный поток, паз обусловливает полный ток. При этом глубина паза меняется существенно - от 45мм в машинах меньшей мощности (600 кВт) до 70мм - в более мощных.

Такие геометрические соотношения пазов и диапазон изменения частоты вращения ротора с изменением мощности генераторов обусловливают особенности возможных вариантов обмоток: могут применяться всыпные обмотки, состоящие из нескольких параллельных ветвей в фазе и нескольких параллельных проводников в секции - для уменьшения диаметра элементарного проводника, учитывая малую ширину паза. $\mathrm{C}$ увеличением числа параллельных ветвей в фазе увеличивается и число витков в секции, необходимое для получения напряжения ветви, соответствующего заданному фазному напряжению, а сечение проводника уменьшается, так как уменьшается ток в ветви. Фазная э.д.с. в таких обмотках рассчитывается по известной формуле [3]:

$$
E_{\phi}=4,44 \cdot f \cdot \Phi_{\delta} \cdot W_{\phi} \cdot k_{o}
$$

где $W_{\phi}$ - требуемое число витков в фазе.

По расчетам, в этом случае число витков в секции может оказаться около 0,5 - при мощности 600 кВт и менее - по мере увеличения мощности генераторов. Число витков в секции равное 0,5 означает, что в одном пазу находиться один проводник, поэтому может быть применена 
"меандровая" обмотка, в которой каждая фаза представляет собой один меандр - непрерывной проводник. Отличительной особенностью такой обмотки в отличии от всыпной из нескольких секций в фазе является отсутствие необходимости соединения секций, возможность увеличения коэффициента заполнения паза выше $(0,34-0,36)$ и мощности при заданной плотности тока, уменьшение потерь в обмотке, т.к. в меандре отсутствует одна лобовая часть "условного" витка.

Однако, при указанных размерах пазов малой ширине и большой глубине применение многофазной меандровой обмотки, расположенной в одной плоскости, может сопровождаться технологическими сложениями её изготовления из-за пересечения меандров в лобовой части обмотки (рис.2), особенно в случае применения шины с близкими с пазами размерами. Возможно выполнение такой обмотки кабелями, число которых равно числу фаз, но в этом случае коэффициент заполнения пазов будет меньше, чем в случае применения шины. Для устранения пересечения меандров в лобовых частях трехфазная обмотка может быть выполнена в виде трех независимых меандров, разнесенных по высоте паза в трех плоскостях (рис.5). В этом случае увеличивается глубина пазов и соответственно - высота зубцов.

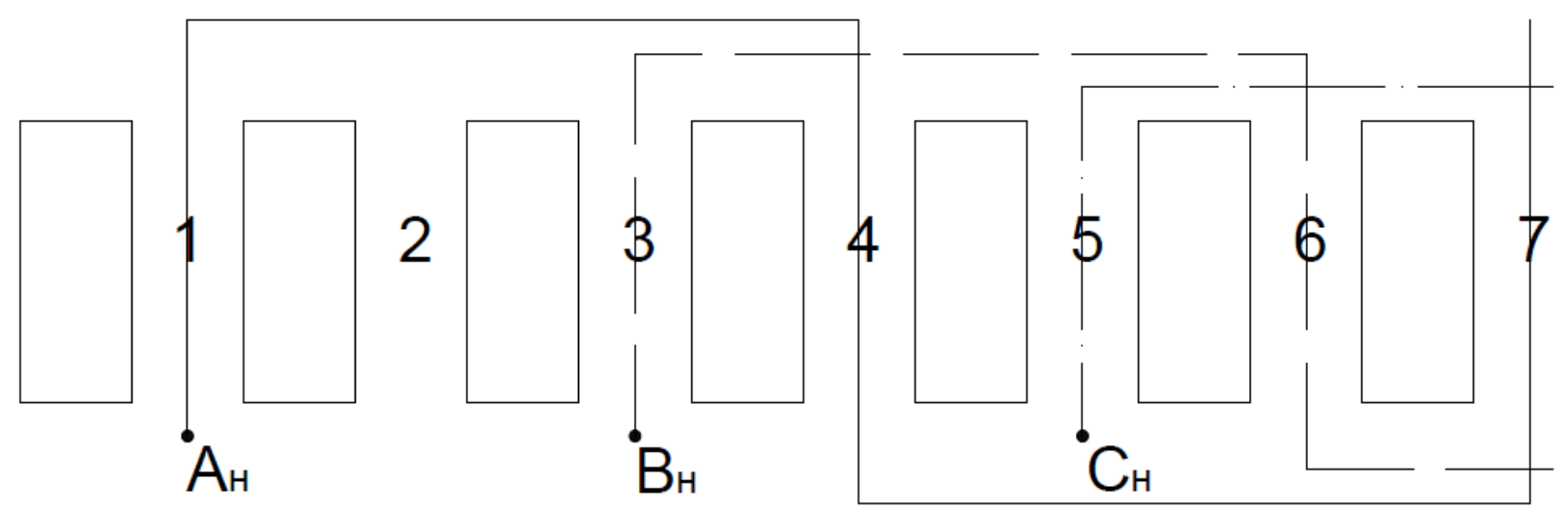

Рис. 2. Трехфазная обмотка из трех меандров, расположенных в одной плоскости.

Fig. 2. Three phase windings made of three meanders, which are located in one plane.

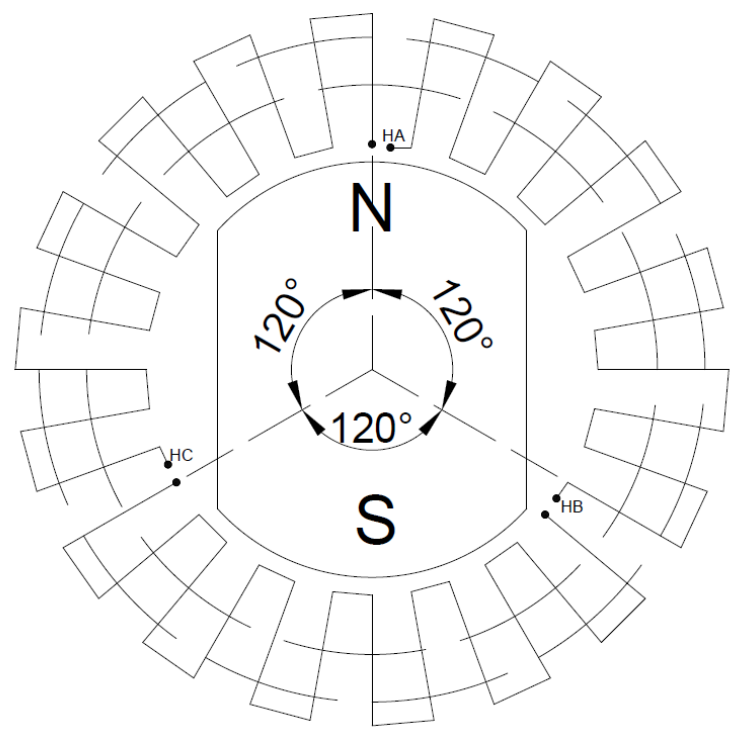

Рис. 3. Трехфазная меандровая обмотка из трех меандров в одной плоскости, каждый из которых занимает треть окружности.

Fig. 3. Three phase meander winding consists of 3 meanders located in one plane, each occupy the third of circle. 

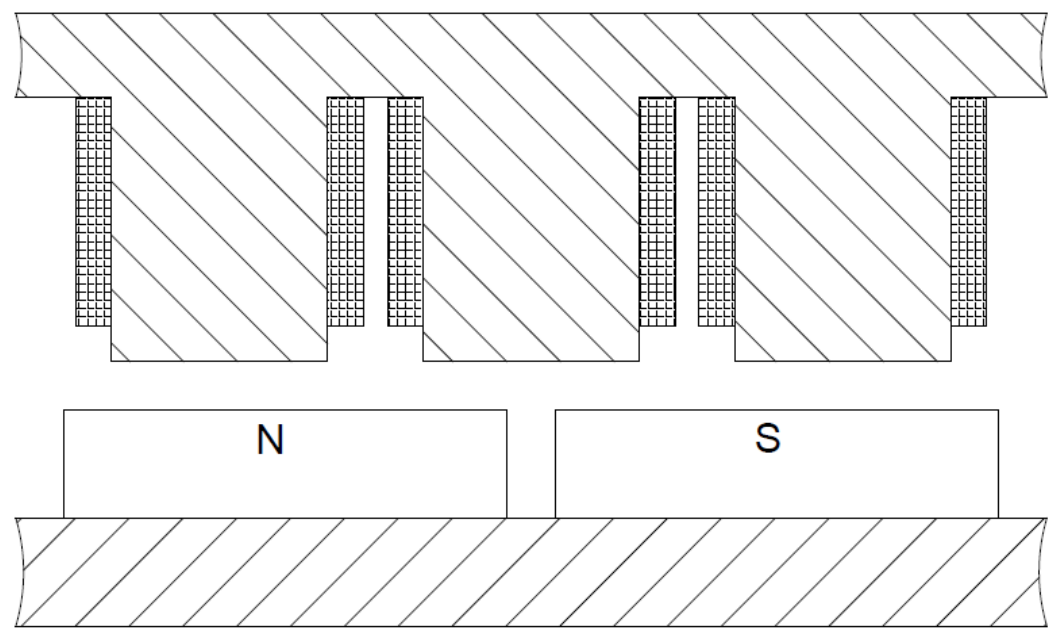

Рис. 4. Трехфазная “катушечная' обмотка.

Fig. 4. Three phase 'coil' winding.

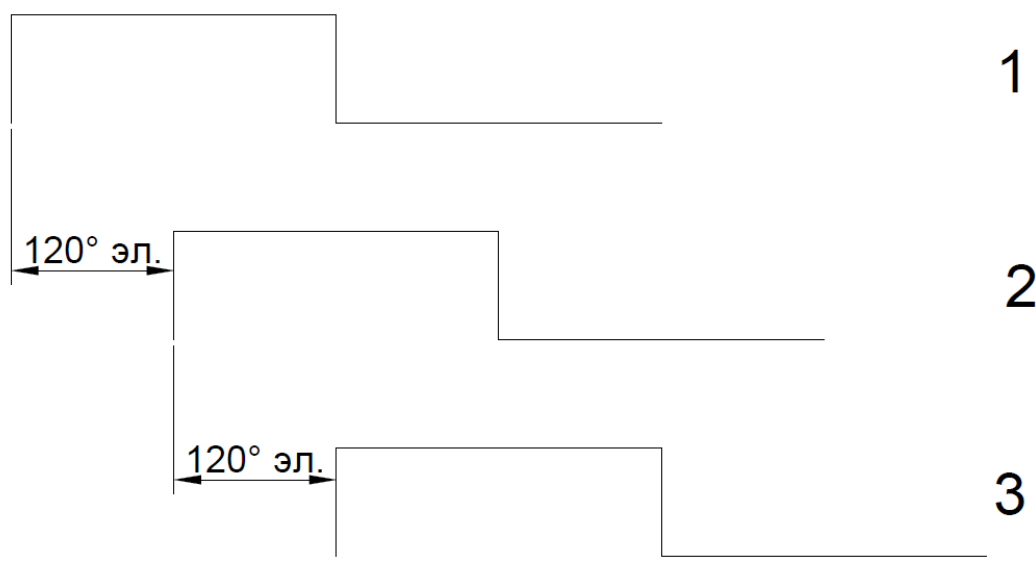

Рис. 5. Трехфазная обмотка в виде трех меандров, расположенных в трех плоскостях по глубине паза.

Fig. 5. Three phase winding of three meanders which are located in three planes in depth of slot.

Зубцы в рассматриваемых машинах являются наиболее насыщенными участками магнитной цепи, потери в них пропорциональны квадрату индукции и массе зубцов и могут составлять около $4.0 \%$ суммарных потерь, поэтому уменьшается мощность. Исключить увеличение потерь в зубцах и пересечения меандров в лобовых частях возможно также в варианте обмотки (рис.3), где меандры расположены в одной полости и каждый из них занимает треть окружности при трехфазном исполнении. Однако при заданной частоте выходного напряжения (6) и определенной частоте вращения ветроколеса (3), которая в безредукторной установке равна скорости вращения генератора, число полюсов в каждой фазе этой обмотки должно быть равно числу полюсов в фазе обмотки, распределенной по всей окружности (рис. 2). По этой причине полюса в пределах каждой фазы обмотки имеют соответственно меньшие размеры, поэтому больше поток рассеяния относительно полезного потока и в результате мощность генератора в этом варианте примерно на треть меньше, чем в ранее рассмотренных.

Как указывалось, число витков в секции при расчете по формуле (9) изменяется от 0,5 - при мощности генератора 600 кВт и уменьшается по мере её увеличения, поэтому расчет генератора с меандровой обмоткой требует уточнения алгоритма расчета, в частности, расчета э.д.с. фазы, которая может быть определена по формуле:

$$
E_{n p}=B_{\delta} \cdot l_{\phi} \cdot v,
$$

где $B_{\delta}=\sqrt{\frac{2}{T} \int_{0}^{T / 2} B_{x}^{2} d x}-$ действующее значение индукции; $\quad v=\pi \cdot D \cdot n-$ окружная скорость 
ротора; $l_{a \phi}-$ активная длина проводников фазы без учета лобовых частей обмотки.

Далее алгоритм расчета генератора с меандровой обмоткой может быть построен в следующей последовательности:

-по результатам, полученным в (1) -(8), определяется полное число пазов $\sum z$, число пазов в фазе при трехфазной обмотке в роторе (8):

$$
z_{\phi}=\frac{\sum z}{3}
$$

- активная длина проводников фазы:

$$
l_{a \phi}=z_{\phi} \cdot l_{c p} ;
$$

- окружная скорость ротора:

$$
v=R \cdot \omega
$$

- э.д.с. фазы:

$$
E_{\phi}=B_{\delta} \cdot l_{a \phi} \cdot v,
$$

где $B_{\delta}-$ предварительное действие значение индукции $(\approx 0,8 \mathrm{~T})$;

- фазное напряжение:

$$
U_{\phi}=\frac{E_{\phi}}{1,15 \ldots 1,18} ;
$$

-фазный ток:

$$
I_{\phi}=\frac{P}{3 \cdot U_{\phi} \cdot \cos \varphi} \approx \frac{P}{3 \cdot U_{\phi} \cdot 0,96}
$$

- сечение фазного меандра (шина, кабель) по заданной плотности тока $\mathrm{j} \approx 4 \div 6 \quad \mathrm{~A} / \mathrm{mm}^{2}$ при естественном охлаждении:

$$
q_{\phi}=\frac{I_{\phi}}{j}
$$

- площадь паза:

$$
S_{n}=\frac{q_{\phi}}{k_{3}} ;
$$

где $k_{3} \approx(0,34 \div 0,6)-$ коэффициент заполнения паза (меньшее значение - для трехфазной обмотки, выполненной кабелем, в которой каждая фаза распределена по всей окружности и все фазы расположены в одной плоскости (рис. 2); большее значение - для аналогичной обмотки, выполненной шиной - при условии решения технологии её изготовления.

Далее расчет машины проводится в соответствии с рекомендациями, приведенными в [4-9]. По рассмотренному алгоритму были проведены расчеты ряда машин, некоторые результаты представлены в таблице 1 на примере

\begin{tabular}{|c|c|c|c|c|c|c|c|c|}
\hline & & I & II & III & IV & V & VI & VII \\
\hline$D$ & $\mathcal{M}$ & 45,5 & 45,5 & 45,5 & 45,5 & 45,5 & 45,5 & 45,5 \\
\hline$\omega$ & $p a d / c$ & 2,52 & 2,52 & 2,52 & 2,52 & 2,52 & 2,52 & 2,52 \\
\hline$n$ & об/мин & 24 & 24 & 24 & 24 & 24 & 24 & 24 \\
\hline$D_{p}$ & $M$ & 3,4 & 3,4 & 3,4 & 3,4 & 3,4 & 3,4 & 3,4 \\
\hline$l_{p}$ & $\mathcal{M}$ & 1,02 & 1,02 & 1,02 & 1,02 & 1,02 & 1,02 & 1,02 \\
\hline $2 p^{\prime}$ & полюсов & 250 & 250 & 250 & 250 & 250 & 250 & 250 \\
\hline$\sum z$ & пазов & 750 & 750 & 750 & 750 & 750 & 750 & 750 \\
\hline$z_{\phi}$ & $\begin{array}{l}\text { пазов в } \\
\text { фазе }\end{array}$ & 250 & 250 & 250 & 250 & 250 & 250 & 250 \\
\hline$l_{a \phi}$ & $\mathcal{M}$ & 254 & 254 & 254 & 254 & 254 & 254 & 254 \\
\hline$v$ & $M / c$ & 4,28 & 4,28 & 4,28 & 4,28 & 4,28 & 4,28 & 4,28 \\
\hline$U_{\text {ф.расч }}$ & $B$ & 740 & 740 & 740 & 740 & 935 & 868 & 740 \\
\hline$P_{\text {э.расч }}$ & $\kappa B m$ & 633 & 633 & 633 & 633 & 859 & 806 & $1790 / 1100$ \\
\hline$\eta$ & o.e. & 0,92 & 0,8 & 0,94 & 0,87 & 0,93 & 0,91 & 0,96 \\
\hline $\cos \varphi$ & o.e. & 0,97 & 0,97 & 0,97 & 0,97 & 0,92 & 0,93 & 0,93 \\
\hline$I_{\phi . p a c u}$ & $A$ & 294 & 294 & 294 & 294 & 333 & 333 & $86,6 / 532$ \\
\hline
\end{tabular}
расчета генератора мощностью 600 кВт с номинальным напряжением 690B, скорости ветра $12,5 \mathrm{~m} / \mathrm{c}$ с разными вариантами обмоток и коэффициентов заполнения пазов $(0,34 ; 0,6)$ в зависимости от типа обмотки.

Таблица 1. Расчетные параметры трехфазного генератора мощности 600кВт при скорости ветра 12,5 м/с.

Table 1. Calculated parameters of three phase $600 \mathrm{~kW}$ generator at the wind speed $12,5 \mathrm{~m} / \mathrm{s}$. 
В таблице 1: $D$ - диаметр ветроколеса; $\omega$ и $n$ - угловая скорость и частота вращения генератора, которая в безредукторном исполнении равна частоте вращения ветроколеса; $D_{p}$ - диаметр ротора генератора; $l_{p}-$ активная длина ротора; $2 p^{\prime}$ - число полюсов; $\Sigma z-$ полное число пазов; $z_{\phi}$ - число пазов в фазе; $l_{a \phi}-$ активная длина всех проводников одной фазы; $v$ - линейная скорость вращения ротора; $U_{\text {ф.расч }}$ расчетное значение фазного напряжения; $P_{\text {эрасч }}$ расчетное значение электромагнитной мощности; $\eta$ - коэффициент полезного действия генератора; $\cos \varphi-$ коэффициент мощности; $I_{\phi . р а с ч}-$ расчетное значение фазного тока.

Варианты I, III - три меандра располагаются в одной плоскости (рис.2); I - коэффициент заполнения паза - 0,34; III - коэффициент заполнения паза 0,64; варианты II, IV - три меандра располагаются в трех плоскостях по высоте паза (рис.5); II - коэффициент заполнения паза 0,34; IV - коэффициент заполнения паза 0,64; варианты V, VI - всыпные обмотки, в которых каждая фаза выполнена из трех параллельных ветвей, а каждая секция - из нескольких параллельных проводников для уменьшения диаметра элементарного проводника, учитывая малую ширину пазов; V число пазов на полюс и фазу - q =1; VI - число пазов на полюс и фазу - q=0,5; VII - меандровая обмотка (рис. 2) с коэффициентом заполнения паза 0,64, но глубина паза равна глубине паза всыпной обмотки с коэффициентом заполнения паза 0,34 (глубокий паз) - для сравнения этого варианта с вариантом V (всыпная обмотка, q=1).

Выводы: 1. С увеличением мощности генератора увеличивается диаметр ротора и при принятом числе пазов на полюс и фазу $(q=0,5 ; 1)$ увеличивается число пазов, но в результате зубцовое деление, определяемое суммой ширины паза и ширины зубца меняется несущественно $\left(t_{z}=b_{z}+b_{n} \approx 15\right.$ мм, при q=1) при значительном изменении глубины паза ( $h_{n}=45 \div 65$ мм); при этом оптимальным по электромагнитным параметрам можно считать равенство $b_{z}=b_{n}$.

2. При уменьшении q в два раза $(1 \div 0,5)$ пропорционально увеличивается ширина паза и зубца, число витков в секции. Увеличение размеров паза и зубца упрощает выполнение обмотки и пакета статора, но при этом уменьшается в 1,07 раза электромагнитная мощность (табл.1) в связи с изменением коэффициента распределения обмотки.

3. Расчет э.д.с. по традиционной формуле (9) показывает изменение числа витков в секции от 0,5 и менее с увеличением мощности машины от 600 кВТ до 3600 кВТ, начиная с фазного напряжения 690В. Число витков в секции, равное
0,5 означает, что в одном пазу находится один проводник. Это обусловило выполнение обмотки в виде меандра, т.е. трехфазная обмотка - это три меандра, выполненные кабелями или шиной.

4. С увеличением мощности генераторов увеличиваются геометрические размеры ротора и статора, число пазов статора и активная длина проводников фазы при меандровой обмотке, т.к. в одном пазу находится один проводник, поэтому увеличивается фазное напряжение.

5.Увеличение коэффициента заполнения паза при принятой плотности тока и расчетном значении фазного тока означает уменьшение площади паза, или увеличение фазового тока за счет увеличения сечения проводников в пазу за счет увеличения размеров паза при меньшем коэффициенте заполнения. Однако, это сопровождается уменьшением сопротивления, ростом тока, потерь в обмотке и в результате превышением допустимых по нагреву удельных потерь с внешней поверхности корпуса генератора, которые по расчетам составляют около 6000 Вт/м² (без торцевых поверхностей генератора модульной конструкции) [1]. По этой причине происходит снижение номинального тока и электромагнитной мощности (табл.1, вариант VII).

6. Расчеты вариантов I, II, III, IV показывают близкие значения электромагнитной мощности, а всыпные обмотки (варианты VI, VII) имеют несколько большую мощность $\left(P_{\ni}\right)$ за счет более высокого расчетного значения фазного напряжения и фазного тока, обусловленных большей площадью паза. В целом рассмотренные варианты обмоток обеспечивают сопоставимые значения электромагнитной мощности, но требуется решение вопросов технологии их изготовления.

7. Возможно применение "катушечных" обмоток (рис.5) при расчетном значении числа витков в секции более 0,5 по формуле (9).

8. При заданных параметрах применение обмотки (рис.4) не целесообразно в виду малой электромагнитной мощности генератора.

1.Кудря С.О., Перминов Ю.Н., Коханевич В.П. Вітроелектрична установка модульной конструкції. Патент на винахід №106673. Україна. МПК Н02К 16/00. Н02К 21/22. № a2013 01473. Заявл. 07.02.13. Опубл. 25.09.14. Бюл. №9.

2. Перминов Ю.Н., Монахов Е.А. Сравнение вариантов конструкций синхронных генераторов с возбуждением от постоянных магнитов для ветроустановок. Відновлювальна енергетика. 2019. № 2. С. 54-60.

3. Костенко М.П., Пиотровский Л.М. Электрические машины, часть вторая. М. Л. 247 с.

4. Мхитарян Н.М., Кудря С.А., Перминов Ю.Н. Проектирование синхронных генераторов для ветроустановок малой мощности. Відновлювальна енергетика. 2006. № 2. С. 40-43.

5. Перминов Ю.Н., Коханевич В.П., Шихайлов Н.А., Перминова С.Ю. Определение параметров и основных размеров генераторов для приливных электростанций малой мощности (до 1000 кВт). Відновлювальна енергетика. 2017. 


\section{№ 3. C. 66-72.}

6. Перминов Ю.Н., Коханевич В.П. Алгоритм расчета синхронных генераторов торцевой конструкции. Відновлювальна енергетика. 2016. №2. С. 45-49.

7. Кудря С.О., Перминов Ю.Н. Некоторые особенности проектирования синхронных ветрогенераторов повышенной мощности с возбуждением от постоянных магнитов. Відновлювальна енергетика. 2014. № 3. С. 54-57.

8. Перминов Ю.Н., Коханевич В.П. Алгоритм расчета синхронных генераторов с возбуждением от постоянных магнитов. Відновлювальна енергетика. 2015. № 2. С. 60-65.

9. Перминов Ю.Н., Монахов Е.А. Разработка методики расчета теплогенератора с постоянными магнитами для ветроустановки. Відновлювальна енергетика. 2017. № 1. C. $56-61$.

\section{REFERENCES}

1. Kudrya S.O., Perminov Yu.M., Kokhanevich V.P. Vitroelektrychna ustanovka modulnoy konstruktsyy. [Windelectric modular set]. Patent. № 106673. Ukraine. IPC H02K 16/00. H02K 21/22. № a 2013 01473. Stated. 07.02.13. Published 25.09.14. Bul. № 9. [in Ukrainian].

2. Perminov Yu.N., Monakhov E.A. Sravnenie variantov konstrukciy sinkhronnikh generator s vozbuzhdeniev ot postoyanikh magnitov dlya vetroustanovok. [Design comparison of synchronous permanent magnet generator for windturbines] Vidnovluvana energetika. 2019. No. 2. Pp. 54-60. [in Russian].

3. Kostenko M.P., Piotrovskiy L.M. Elekticheskie mashini. Chast 2. [Electrical machines. Part 2]. M. L. 247 p. [in Russian].

4. Mkhitarian N.M., Kudrya S.A., Perminov Yu.N. Proektyrovanye synxronnix heneratorov dlya vetroustanovok maloj moshhnosty. [Design of synchronous generators for low power wind turbines]. Vidnovluvana energetika. 2006. No. 2 Pp. 40-43. [in Russian].

5. Perminov Yu.N., Konahevich V.P., Shikhailov N.A., Perminov S.Yu. Opredelenie parametrov i osnovnyikh razmerov generatorov dlya prilivnyih elektrostantsiy maloy moschnosti (do $1000 \mathrm{kVt}$ ). [Determination of parameters and basic dimensions of the generator of low power tidal plants (up to $1000 \mathrm{~kW})$ ]. Vidnovluvana energetika. 2017. No. 3. Pp. 66-72. [in Russian].

6. Perminov Yu.N. Alhorytm rascheta synxronnix heneratorov torcevoj konstrukcyy. [The algorithm for calculating synchronous generators of the end structure]. Vidnovluvana energetika. 2016. No. 2. Pp. 45-49. [in Russian].

7. Kudrya S.A., Perminov Yu.N. Nekotorie osobennosty proektyrovanyya synxronnix vetroheneratorov povishennoj moshhnosty s vozbuzhdenyem ot postoyannix mahnytov. [Some design features of high-power synchronous wind generators with permanent magnet excitation]. Vidnovluvana energetika. 2014 No. 3. Pp. 54-57. [in Russian].

8. Perminov Yu.N., Konahevich V.P. Algoritm rascheta sinhronnyh generatorov $\mathrm{s}$ vozbuzhdeniem ot postojannyh magnitov. [Algorithm for calculating synchronous generators with excitation from permanent magnets]. Vidnovluvana energetika. 2015. No. 2. Pp. 60-65. [in Russian].

9. Perminov Yu.N., Monakhov E.A. Razrabotka metodiki rascheta teplogeneratora $\mathrm{s}$ postojannymi magnitami dlja vetroustanovki. [Development of methods for calculating a permanent magnet heat generator for a wind turbine]. Vidnovluvana energetika. 2017. No. 1. Pp. 56-61. [in Russian]

\section{ОСОБЛИВОСТІ ПРОЕКТУВАННЯ \\ ВІТРОГЕНЕРАТОРІВ ПІДВИЩЕННОӦ ПОТУЖНОСТІ

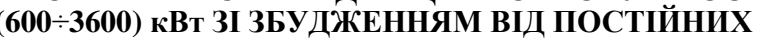 MAГHITIB}

Ю.Н. Перминов ${ }^{1}$, канд. техн. наук, Є.А. Монахов ${ }^{2}$, Л.П. Волков ${ }^{3}$

${ }^{1}$ Інститут відновлюваної енергетики НАН України, 02094, вул. Гната Хоткевича, 20А, м. Киев, Україна.

${ }^{2}$ Національний технічний університет України «Київський політехнічний інститут імені Ігоря Сікорського», 03056, пр. Перемоги, 37, м. Киев, Україна.

${ }^{3}$ Інститут екологічного управління та збалансованого природовикористування,

02002, вул. Нікольсько-Слобідська, 6-Д, м. Киев, Україна.

В основу роботи покладено результати розрахунків ряду синхронних безредукторних вітрогенераторів модульноі конструкиї [1] зі збудженням від постійних магнітів $\mathrm{NdFeB}$ потужністю $(600 \div 3600) \mathrm{\kappa Bm}$ при прийнятій за кордоном розрахункової швидкості вітру - 12,5 м/с. Такий підхід дослідження машин дозволяє оиінити складний взаємозв'язок їх параметрів при мінімумі припущень, зокрема, врахувати насичення найбільш навантажених ділянок магнітного кола $i$ визначити доиільність застосування деяких можливих варіантів обмоток, які забезпечують найбільшу електромагнітну потужність генераторів з урахуванням технологічності їх виконання; показані особливості зміни зубиевої зони машин підвищеноі потужності, які зумовлені низькою швидкістю обертання генераторів в безредукторних установках і обтрунтована 3 цієї причини можливість застосування "меандрової" обмотки.

У такій обмотиі кожна фаза виконується у вигляді одного безперервного провідника, що дозволяє виключити з'єднання секиій у фазі, що має місие в традииійних обмотках, зменшити опір обмотки завдяки відсутності однієї лобовоі частини в порівнянні з витком секиії. При виріменні технології виготовлення таких обмоток у вигляді шини можливе підвитення коеріцієнта заповнення пазів потужності генератора в певних габаритах. Можливо виготовлення такої обмотки кабелями, в иьому випадку трифазна обмотка представляс три кабелі, що дозволить спростити ї̈ виготовлення, але коефічієнт заповнення пазів буде менше.

В роботі проведено порівняння різних варіантів меандрових обмоток з метою спрощення їх виготовлення і традииійних всипних обмоток з різною кількістю пазів на полюс і фазу з метою збільшення зубцевого кроку $i$ спрощення виготовлення пакета статора $і$ обмотки. Бібл. 9, табл. 1 , рис. 5 .

Ключові слова: безредукторні генератори модульноі конструкиї; варіанти обмоток; зубиева зона; "меандрова обмотка"; коефіиієнт заповнення пазів; число пазів на полюс $i$ фазу; число витків в секиії; активна довжсина провідників; електромагнітна потужність. 